\title{
Targeting rheumatoid inflammation and joint destruction in the mouse
}

\author{
Masaki Inada and Stephen M. Krane \\ Department of Medicine, Harvard Medical School, and the Center for Immunology and Inflammatory Diseases, Massachusetts General Hospital,
} Boston, Massachusetts, USA

J. Clin. Invest. 110:611-612 (2002). doi:10.1172/JCI200216549.

For over four decades, evidence has accumulated indicating a role for prostaglandins (PGs) in the pathogenesis of rheumatoid arthritis (1). Nevertheless, although some patients with rheumatoid arthritis may demonstrate considerable benefit from nonsteroidal anti-inflammatory drugs (2), the striking effects observed in animal models are not usually observed in most patients. Furthermore, the more specific inhibitors of cyclooxygenase- 2 (COX-2), which have less serious gastrointestinal toxicity, have not been shown to have greater efficacy than the older, less specific drugs $(3,4)$. Several possible explanations for relatively weak clinical responses in patients with rheumatoid arthritis contrasted with strong effects in animal models of arthritis have been proposed. Recent findings of subtypes of prostaglandin E2 (PGE2) receptors encoded by different genes with different tissue distribution and coupled to different intracellular signaling events (5-7) have now suggested the means to take a new look at how PGs could function in joint inflammation.

In this issue of the JCI, McCoy et al. (8) report on having used mice with

\footnotetext{
Address correspondence to: Stephen M. Krane, Department of Medicine, and the Center for Immunology and Inflammatory Diseases, Massachusetts General Hospital, 149 13th Street, Charlestown, Massachusetts 02129, USA. Phone: (617) 726-5650;

Fax: (617) 726-5651;

E-mail: krane.stephen@mgh.harvard.edu. Conflict of interest: No conflict of interest has been declared.

Nonstandard abbreviations used: prostaglandin (PG); cyclooxygenase (COX); prostaglandin E2 (PGE2); collagen antibodyinduced arthritis (CAIA); parathyroid hormone (PTH).
}

targeted mutations in each of the PGE receptors so far identified, EP1, EP2, EP3 and EP4, to take a deeper look into possible functions of PGE2 in joint inflammation. They employed a collagen antibody protocol to induce arthritis (CAIA) in mice, producing clinically evident disease within a few days of injection and major joint pathology by 10 days. In this system, EP4-/- mice showed a profound decrease in the intensity of the inflammation, as well as a decrease in markers of joint destruction, compared to that in the other receptor null mice, clearly indicating the importance of the EP4 receptor in the pathogenesis of collagen antibody-induced arthritis (CAIA).

Although the attenuated inflammatory response in $\mathrm{EP}^{-/-}$mice is clear, evidence for the protective effects of the mutation EP4 on damage to joint structures is less compelling. The advantage of using a mouse model with rapid onset of arthritis, as contrasted with the standard, more chronic model of type II collagen-induced arthritis, is to some extent offset by the lesser degree of cartilage and bone damage in the CAIA protocol and the paucity of erosions characteristic of progressive human rheumatoid arthritis. Here, the authors followed damage to articular cartilage by Safranin Red staining for proteoglycans and immunostaining using a monoclonal antibody that detects an epitope in an unfolded cleavage fragment of type II collagen, and they state that bone destruction was reduced specifically in EP4-/- animals.

Indirect support for this conclusion comes from earlier studies $(9,10)$ of $\mathrm{EP}$ receptor function during bone remodeling, employing agonists spe- cific for each EP subtype or targeted EP receptor mutation. Ex vivo bone resorption by cultured, wild-typemouse calvariae has been found to be markedly stimulated by either PGE2 or an EP4 agonist, but only slightly by an EP2 agonist and not at all by EP1 and EP3 agonists. The enhanced bone resorption correlates with cAMP production and an increase in the population of large, tartrate-resistant acid phosphatase-positive osteoclasts. It is also accompanied by increased expression of matrix metalloproteinases such as MMP-2 and MMP-13, and of the receptor activator of $\mathrm{NF}-\mathrm{\kappa B}$ ligand, which is associated with increased osteoclast formation and activity. In studies of mice with a targeted null mutation in each of the four EP subtypes, only mice lacking the EP4 subtype had blunted boneresorptive responses to PGE2; as would be predicted, these mutant mice retained the normal response to dibutyryl cyclicAMP, which bypasses signaling through the PGE receptors. Bone resorption in response to inflammatory cytokines and other ligands that act through PGE2 production were also blunted in $\mathrm{EP}^{-/-}$mice.

Thus, the EP4-mediated bone resorption observed by McCoy et al. (8) may result from local inflammation. Indeed, experiments using PGG/H synthase-2-deficient (Cox2---) mice (11) have shown that normal activity of this enzyme and, therefore, normal responses generating PGE2 are required for stimulation of bone resorption by parathyroid hormone $(\mathrm{PTH})$ and $1,25(\mathrm{OH})_{2}$ vitamin $\mathrm{D}$. Although the latter molecules are usually not grouped among inflammatory cytokines, $1,25(\mathrm{OH})_{2}$ vitamin 
$\mathrm{D}$ is produced by immune cells, and the presence of parathyroid hormone-related protein has been demonstrated in cellular infiltrates in rheumatoid joints (12).

Skeletal actions of PGE2 are not limited to stimulating bone resorption; PGE2 can also induce bone formation in humans and other animals. Of particular interest is the finding that PGE2 induces increased bone formation in EP1-, EP2- and EP3-deficient, but not EP4-deficient, mice and that only an EP4-selective agonist can induce bone formation in wild-type mice (13). A selective EP4 agonist also prevents the bone loss that follows ovariectomy. Osteoblast differentiation and bone nodule formation in bone marrow stromal cell cultures is diminished in Cox $2^{-/-}$mice and is correlated with reduced expression of two genes critical for osteoblast function, Cbfa1 and Osterix (14). Thus, as has also been seen for PTH, dosage and scheduling may determine whether bone resorption or bone formation predominates following treatment with EP4 agonists.

All of these observations point to a major role for PGE2 in inflammatory responses and skeletal remodeling events in mice, but we need to under- stand why this role does not appear to be as impressive in human rheumatoid arthritis. Is inhibition of prostaglandin synthesis simply incomplete at current recommended dosages of COX-2 antagonists or the older nonsteroidal anti-inflammatory drugs? Do other, as-yet-unidentified ligands act on EP receptors in the absence of prostaglandins? Would the therapeutic effects of EP4 antagonists be more evident than the therapeutic effects of COX-2 inhibitors in rheumatoid arthritis? A facile reference to "species differences" will not help here. Informative, creative experiments like those in the present work and the others noted will be needed to resolve the apparent discrepancies and provide useful therapies for human arthritis.

1. Robinson, D.R., Dayer, J.-M., and Krane, S.M 1979. Prostaglandins and their regulation in rheumatoid arthritis. Ann. N.Y. Acad. Sci. 332:279-294.

2. Cush, J.J., et al. 1990. Correlation of serologic indicators of inflammation with effectiveness of nonsteroidal antiinflammatory drug therapy in rheumatoid arthritis. Arthritis Rheum. 33:19-28.

3. Simon, L.S., et al. 1999. Anti-inflammatory and upper gastrointestinal effects of celecoxib in rheumatoid arthritis: a randomized controlled trial. JAMA. 282:1921-1928.

4. Bombardier, C., et al. 2000. Comparison of upper gastrointestinal toxicity of rofecoxib and naproxen in patients with rheumatoid arthritis. N. Engl. J. Med. 343:1520-1528.
5. Nguyen, M., et al. 1997. The prostaglandin receptor EP4 triggers remodelling of the cardiovascular system at birth. Nature. 390:78-81.

6. Ushikubi, F., et al. 1998. Impaired febrile response in mice lacking the prostaglandin $\mathrm{E}$ receptor subtype EP3. Nature. 395:281-284.

7. Tilley, S.L., et al. 1999. Reproductive failure and reduced blood pressure in mice lacking the EP2 prostaglandin E2 receptor. J. Clin. Invest. 103:1539-1545.

8. McCoy, J.M., Wicks, J.R., and Audoly, L.P. 2002. The role of prostaglandin E2 receptors in the pathogenesis of rheumatoid arthritis. J. Clin. Invest. 110:651-658. doi:10.1172/JCI200215528.

9. Suzawa, T., et al. 2000. The role of prostaglandin E receptor subtypes (EP1, EP2, EP3, and EP4) in bone resorption: an analysis using specific agonists for the respective EPs. Endocrinology. 141:1554-1559.

10. Miyaura, C., et al. 2000. Impaired bone resorption to prostaglandin E2 in prostaglandin $\mathrm{E}$ receptor EP4-knockout mice. J. Biol. Chem. 275:19819-19823.

11. Okada, Y., et al. 2000. Prostaglandin G/H synthase- 2 is required for maximal formation of osteoclast-like cells in culture. J. Clin. Invest. 105:823-832.

12. Funk, J.L., et al. 1998. Synovium as a source of increased amino-terminal parathyroid hormonerelated protein expression in rheumatoid arthritis. A possible role for locally produced parathyroid hormone-related protein in the pathogenesis of rheumatoid arthritis. J. Clin Invest. 101:1362-1371.

13. Yoshida, K., et al. 2002. Stimulation of bone formation and prevention of bone loss by prostaglandin E EP4 receptor activation. Proc. Natl. Acad. Sci. USA. 99:4580-4585.

14. Zhang, X., et al. 2002. Cyclooxygenase-2 regulates mesenchymal cell differentiation into the osteoblast lineage and is critically involved in bone repair. J. Clin. Invest. 109:1405-1415. doi:10.1172/JCI200215681. 